\title{
The sound of getting rid of coronavirus by RNA interference technology: RNAi against COVID-19
}

\author{
Ali Ahmadi ${ }^{1}$, Hamed Hekmatnezhad ${ }^{2,}{ }^{*}$ \\ ${ }^{1}$ Faculty of Biological Sciences and Technologies, Sari Branch, Islamic Azad University, Sari, Iran \\ ${ }^{2}$ Department of Basic Sciences, Sari Agricultural Sciences and Natural Resources University, Sari, Iran
}

Keywords: RNA interference, siRNA, COVID-19, SARS-CoV-2

Coronaviruses are a large family of Coronaviridae and a subfamily of Coronavirinae and the order Nidovirales that range from the common cold virus to the cause of more serious illnesses such as severe acute respiratory syndrome (SARS), middle east respiratory syndrome (MERS), and severe acute respiratory syndrome coronavirus 2 (SARS-CoV-2). Spherical or pleomorphic enveloped particles containing singlestranded RNA (ssRNA) are associated with a nucleoprotein within a capsid comprised of matrix protein. The envelope bears club-shaped glycoprotein projections [1]. Coronavirus disease 2019 (COVID-19), reported on December 31, 2019, in Wuhan, China, causes the acute respiratory syndrome. To date, thousands of people around the world are infected with the virus every day, while the number of people who die by COVID-19 is also significant. COVID-19 disease may manifest either as an asymptomatic infection or a mild to severe pneumonia [2]. Due to the epidemic of COVID-19 virus in the world, there is an urgent need for new antiviral [3]. To date, there is no known definitive cure for coronavirus, and no vaccine has been developed that is approved by the World Health Organization (WHO). Research to date has shown that the SARS-CoV-2 and SARS-CoV genes are up to $79 \%$ identical sequence and their receptorbinding domain structure are very similar [4]. Both viruses have an animal reservoir and have been transmitted from animal to human. SARS was first reported in the Guangdong province of southern China in 2002 [5].

As an effort to prevent and inhibit COVID-19 virus replication, RNA interference (RNAi) technology can be considered. This technology has been previously used to turn off the expression of the virus gene in SARS-CoV, HIV, HCV, and HBV [6]. RNA interference is a process in which double-stranded RNA causes a specific sequence in homologous genes to be silenced. This natural mechanism for silencing specific sequence genes has given biologists hope in the treatment of many diseases, including viral diseases and cancer, and may have important practical applications in agriculture, functional genomics, and therapeutic interventions. Historically, RNAi has been identified with terms such as repressing or silencing genes after transcription. The RNAi mechanism is evolutionarily conserved and was found in a wide range of eukaryotic organisms [7]. The mechanism of RNAi can be accomplished in three ways, including synthetic short-interference RNAs (siRNAs; 19-27 long double-stranded nucleotide RNAs) as well as in situ production of short hair RNAs (shRNAs) or pointed through plasmid DNA-based expression vectors (pDNA). The process of gene deactivation by

\footnotetext{
*Corresponding author:

Hamed Hekmatnezhad, MSc

Department of Basic Sciences, Sari Agricultural Sciences and

Natural Resources University, Sari, Iran

Tel/Fax: +98911 9915699

Email: hamedhekmatnejad@yahoo.com

https://orcid.org/oooo-0002-5716-9240
}

Received: November, 4, 2020

Accepted: November, 5, 2020
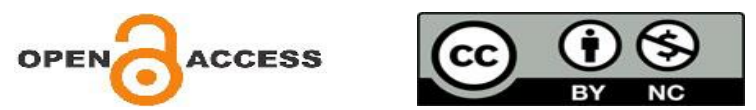
RNAi mainly mediated through two small ribonucleic acid molecules called microRNA (miRNA) and small interfering RNA (siRNA). Specific inhibition of cellular mRNA in mammalian cells performed by the introduction of synthetic 21- to 23-nucleotide duplexes of RNA. Thus, RNAi specifically inhibits gene expression and viral genome replication in the host cell $[8,9]$. The RNAi mechanism inhibits gene expression in many eukaryotes, including animals. In this process, when a double-stranded RNA enters the cytoplasm, it is detected by the Dicer enzyme, and fragments of 21 to 25 nucleotides, known as siRNAs, are produced by the enzyme [10]. Then, these short two-string pieces will follow different destinies. They may be attached to the RNA-induced silencing complex (RISC) set and become single-stranded, with one strand separated from the whole set by one of the subunits and then attached to complementary versions and cut by the RISC set. Cleaves transcripts will be degraded by cytoplasmic RNases [11]. Good results have been obtained in the treatment of SARS virus using RNAi, which can be extended to other viruses of the coronavirus family. The RNAi strategy has been proven to work well in inhibiting different types of virus infections such as rotavirus [12], influenza virus [13], and HIV-1 [14-15]. In recent years, many studies and researches have been done in the treatment of SARS through siRNA. The results of most of these studies show that all siRNA duplexes specifically reduce the expression of the SARS-CoV gene to varying degrees compared to the control. Also, it was well demonstrated that DNA vector-based siRNA can effectively and specifically inhibit the expression of the spike protein gene in SARS-CoV cells and inhibit virus pathogenicity [6].

These results suggest that RNAi, as an effective antiviral strategy, may be used to inhibit COVID-19 infection, as previously demonstrated in similar viral infections such as SARS, MERS, etc. Since the coronavirus is very similar gnomically and structurally to the SARS virus, it is to be hoped that RNAi technology can be used to control the virus and prevent it from becoming pathogenic in humans, thus ending this bitter nightmare. Various cellular functions, including control of gene expression, chromatin status change, and gene silencing, are controlled by RNAi pathways. Speed, accuracy, economy, targeted transmission, and minimal toxicity are some of the factors affecting the therapeutic properties of RNAi. Access to methods to identify, design, and proprietary pharmaceuticals in the process of RNAi, can be considered for the next generation of drugs and can also be an effective treatment for emerging viral diseases such as COVID19.

\section{Author Contributions}

All authors contributed equally to this manuscript, and approved the final version of manuscripts.

\section{Conflict of Interests}

The authors declare that they have no conflicts of interest.

\section{Ethical declarations}

Not applicable.

\section{Financial Support}

None.

\section{References}

1. Chen Y, Liu Q, Guo D. Emerging coronaviruses: Genome structure, replication, and pathogenesis. J Med Virol. 2020; 92(4):418-23.

2. Lundstrom K. Coronavirus Pandemic-Therapy and Vaccines. Biomedicines. 2020; 8(5).

3. Lu R, Zhao X, Li J, Niu P, Yang B, Wu H, et al. Genomic characterisation and epidemiology of 2019 novel coronavirus: implications for virus origins and receptor binding. Lancet. 2020; 395(10224):565-74.

4. Zhou P, Yang XL, Wang XG, Hu B, Zhang L, Zhang W, et al. A pneumonia outbreak associated with a new coronavirus of probable bat origin. Nature. 2020; 579(7798):270-3.

5. Zhu H, Wang L, Fang C, Peng S, Zhang L, Chang G, et al. Clinical analysis of 10 neonates born to mothers with 2019-nCoV pneumonia. Transl Pediatr. 2020; 9(1):51-60. 6. Zhang Y, Li T, Fu L, Yu C, Li Y, Xu X, et al. Silencing SARS-CoV Spike protein expression in cultured cells by RNA interference. FEBS Lett. 2004; 560(1-3):141-6.

7. Kurreck J. RNA interference: from basic research to therapeutic applications. Angew Chem Int Ed Engl. 2009; 48(8):1378-98.

8. Elbashir SM, Lendeckel W, Tuschl T. RNA interference is mediated by 21- and 22-nucleotide RNAs. Genes Dev. 2001; 15(2):188-200.

9. Zamore PD, Tuschl T, Sharp PA, Bartel DP. RNAi: doublestranded RNA directs the ATP-dependent cleavage of mRNA at 21 to 23 nucleotide intervals. Cell. 2000; 101(1):25-33.

10. Taylor DW, Ma E, Shigematsu H, Cianfrocco MA, Noland CL, Nagayama K, et al. Substrate-specific structural rearrangements of human Dicer. Nat Struct Mol Biol. 2013; 20(6):662-70.

11- Shi Y. Mammalian RNAi for the masses. Trends Genet. 2003; 19(1):9-12. 
Ahmadi et al.

12. Déctor MA, Romero P, López S, Arias CF. Rotavirus gene silencing by small interfering RNAs. EMBO Rep. 2002; 3(12):117580.

13. Ge Q, McManus MT, Nguyen T, Shen CH, Sharp PA, Eisen HN, et al. RNA interference of influenza virus production by directly targeting mRNA for degradation and indirectly inhibiting all viral RNA transcription. Proc Natl Acad Sci U S A. 2003; 100(5):2718-
14. Coburn GA, Cullen BR. Potent and specific inhibition of human immunodeficiency virus type 1 replication by RNA interference. J Virol. 2002; 76(18):9225-31.

15. Martínez MA, Clotet B, Esté JA. RNA interference of HIV replication. Trends Immunol. 2002; 23(12):559-61. 